\title{
Percepção de auto-eficácia e auto-relato de falhas de memória prospectiva e retrospectiva ${ }^{1}$
}

\author{
Daniela Benites \\ Sidia M. Callegari Jacques \\ Gustavo Gauer \\ William B. Gomes \\ Universidade Federal do Rio Grande do Sul
}

\begin{abstract}
RESUMO
A percepção de auto-eficácia geral em jovens e idosos serviu de base para o estudo das diferenças entre o auto-relato de falhas de memória prospectiva e retrospectiva. Uma amostra de 642 participantes, com idade entre 16 e 81 anos $(26,62 \pm 13,89)$, respondeu ao Questionário Sociodemográfico, à Escala de Auto-eficácia Geral Percebida (EAEGP), e ao Questionário de Memória Prospectiva e Retrospectiva (PRMQ-10). De acordo com os resultados obtidos, auto-eficácia geral apresentou maior correlação com o auto-relato de falhas de memória prospectiva, enquanto idade e escolaridade correlacionaram com o auto-relato de falhas de memória retrospectiva. Como resultado adicional, verificou-se que existem pontos de reciprocidade entre auto-relato de falhas de memória e resultados de desempenho em tarefas de memória. Tais resultados assinalam maior vinculação entre as dimensões de auto-eficácia e a memória prospectiva.
\end{abstract}

Palavras-chave: auto-eficácia; memória; auto-relato

\begin{abstract}
Perception of self-efficacy and self-report of prospective and retrospective memory failures

The general perception of self-efficacy in young and old people was taken as the basis for the study of the differences among self-reported prospective and self-reported retrospective memory. The participants were 642 aged between 16 and 81 years old $(26,62 \pm 13,89)$, who have answered the Sociodemographic Questionnaire, the General Perception of Self-efficacy Scale (GPSES), and the Prospective and Retrospective Memory Questionnaire (PRMQ-10). Self-efficacy showed higher correlation with self-reported prospective memory failures while years of formal education and age correlated with self-reported retrospective memory failures. As a further result it was verified reciprocity between self-reported memory failures and performance in memory tasks, suggesting a strong association between the dimensions of self-efficacy and prospective memory.
\end{abstract}

Keywords: self-efficacy; memory; self-report

Falhas de memória são mais preocupantes entre os idosos, em consequiência do declínio cognitivo que ocorre com o passar dos anos. As falhas podem estar vinculadas a um baixo nível de auto-eficácia de memória (Comijs, Deeg, Dik, Twisk \& Jonker, 2002), ou indicarem processos degenerativos como Declínio Cognitivo Leve, Alzheimer e Parkinson.

Auto-eficácia de memória é um dos aspectos da meta-memória e designa um sistema de crenças dinâmico, auto-avaliativo com relação à competência e confiança na memória pessoal (Eccles \& Wigfield, 2002). A teoria da auto-eficácia estabelece que ações e emoções induzidas por determinadas situações são parcialmente mediadas pela percepção de auto-eficácia (Bandura, 1977). As crenças de auto-eficácia determinam sentimentos, pensamentos e comportamentos por meio da sua influência nos processos cognitivos, motivacionais, afetivos e na seleção de ambientes (Bandura, 1994). O conceito de auto-eficácia já foi investigado nas suas interações com demandas sociais implícitas (Telch \& Bandura, 1982), processos cognitivos (Bandura, 1989), promoção de saúde (Bandura, 1998), escolha vocacional (Bandura, Barbaranelli, Caprara \& Pastorelli, 2001), e nas relações com a teoria de locus de controle (Bandura \& Locke, 2003).

Pesquisas demonstraram que idosos tendem a ter auto-eficácia de memória mais baixa do que adultos 
jovens (Hertzog, Dixon, Schulenberg \& Hultsch, 1987), fato que pode estar relacionado a uma imagem social negativa do envelhecimento (Levy, 1996). A auto-eficácia de memória parece influenciar positivamente as habilidades de memória (Berry, West \& Dennehey, 1989; Carneiro \& Falcone, 2004; McDougall \& Kang, 2003; Seeman, Rodin \& Albert, 1993; Welch $\&$ West, 2005), pois quanto maior a auto-eficácia de memória de um indivíduo, tanto melhor suas habilidades nas tarefas que envolvem a memória.

Queixas de memória podem estar relacionadas à baixa auto-eficácia de memória, porém não ao reduzido desempenho de memória (Comijs \& cols., 2002). A investigação da percepção de idosos sem declínio cognitivo sobre aspectos de personalidade, saúde física e afetividade, encontrou uma associação entre baixa auto-eficácia e desempenho de memória, com sintomas de ansiedade, e entre nível de escolaridade e queixas de memória. Esses resultados indicaram que pessoas com mais anos de escolaridade tendem a perceber melhor a mudança de qualidade no desempenho cognitivo e que queixas de memória podem refletir um estado geral de bem-estar diminuído. Essas pesquisas investigaram somente a memória retrospectiva (MR), memória utilizada em situações relacionadas a fatos passados, tais como recordar o nome alguém, reconhecer um lugar. Não consideraram, portanto, a memória prospectiva (MP), ou de intenção (Smith, Sala, Logie \& Maylor, 2000) que é utilizada na realização de atividades no futuro associadas a intenções prévias (Marsh, Hicks \& Watson, 2002). Um exemplo de memória prospectiva seria lembrar de pegar o filho na escola ao final do dia.

Entre os poucos estudos de auto-eficácia de memória que avaliaram a memória prospectiva e retrospectiva, está o de McDonald, Gould e Tychynski (1999). $\mathrm{O}$ estudo mostrou a influência significativa da autoeficácia de memória sobre o desempenho de memória prospectiva e a ausência dessa influência sobre o desempenho da memória retrospectiva. Ainda, percepções de melhor capacidade de memória, estabilidade, e ansiedade associaram-se com melhor desempenho em tarefas de memória prospectiva baseadas no tempo, ou seja, naquelas em que a lembrança da intenção ocorre após certo período. Tais resultados indicam que tarefas de memória prospectiva devem ser incluídas em investigações sobre o papel das crenças no desempenho de idosos.

Para Bandura (1989), a auto-eficácia é sempre específica, ou seja, relacionada diretamente a determinado domínio ou situação particular de funcionamento, como a memória. Para Scholz, Doña, Sud e
Schwarzer (2002), a auto-eficácia pode ser geral e referir-se a uma autoconfiança global na habilidade pessoal sobre um grande número de demandas ou situações (Sherer \& Maddux, 1982; Scholz \& cols., 2002). Esses autores concordam com o postulado inicial de Bandura, mas apresentam um modelo em que o grau de especificidade ou generalidade varia segundo o contexto, correspondendo a uma confiança global em habilidades pessoais sobre um grande número de situações. Ao aumentar-se o nível de generalidade das perguntas sobre a auto-eficácia nos questionários desse construto, foi possível examinar um amplo conjunto de diferentes crenças (Scholz \& cols., 2002).

O modelo de auto-eficácia geral foi desenvolvido e viabilizado para pesquisas através da Escala de AutoEficácia Geral Percebida (EAEGP) por Matthias Jerusalem e Ralf Schwarzer, em 1979. A escala consistia em um instrumento com 20 itens. Em 1981 o número de itens foi reduzido para 10 e a escala foi adaptada para 28 idiomas. Posteriormente, Scholz e cols. (2002) investigaram as propriedades psicométricas e avaliaram o construto de auto-eficácia geral em 25 países. A tradução do instrumento para várias línguas foi sensível à cultura e a estrutura da escala foi conservada. No Brasil, a EAEGP (Scholz \& cols., 2002) foi traduzida e validada por Teixeira e Dias (2005).

Estudos sobre a readaptação de indivíduos que passaram por longos períodos de estresse apontaram que a auto-eficácia geral influencia na retomada do curso de vida (Cavanaugh \& Green, 1990). Pacientes cardíacos que obtiveram escores altos em auto-eficácia geral apresentaram melhor recuperação uma semana após a cirurgia e melhor qualidade de vida um ano mais tarde, em comparação com pacientes com baixa auto-eficácia geral (Schröder, Schwarzer \& Konertz, 1998). Um estudo com refugiados alemães revelou que os indivíduos com alto nível de autoeficácia geral eram mais saudáveis, melhor integrados socialmente e mais freqüentemente empregados dois anos após a transição estressante do que o grupo com baixo nível de auto-eficácia geral (Schwarzer, Hahn \& Jerusalem, 1993).

Sabe-se que as queixas de falhas de memória podem estar relacionadas à baixa auto-eficácia de memória e não ao desempenho real (Comijs \& cols., 2002), e que a auto-eficácia de memória parece influenciar somente o desempenho de memória prospectiva, e não da memória retrospectiva (McDonald, Gould \& Tychynski, 1999). Sendo-se assim, pergunta-se qual as interações entre auto-eficácia geral e o auto-relato de falhas de memória prospectiva e retrospectiva? $\mathrm{O}$ 
presente estudo analisa a interferência de fatores sociodemográficos e do nível de auto-eficácia geral no autorelato de falhas de memória geral, prospectiva e retrospectiva, segundo o PRMQ-10, versão em português (Benites \& Gomes, prelo; Smith \& cols., 2000). Investiga, igualmente, a variância no auto-relato das falhas de memória entre grupo de idosos e não idosos e segundo o nível de auto-eficácia (baixa e alta).

\section{MÉTODO}

\section{Participantes}

A amostragem foi intencional e ficou composta por 642 participantes, com idade entre 16 e 81 anos (mé- dia \pm DP: $26,6 \pm 13,9$ anos), com 4 a 25 anos de educação formal $(13,8 \pm 3,0)$, sendo a maioria do sexo feminino $(63 \%)$, solteiros $(77,9 \%)$, de nível socioeconômico médio. Para a análise dos dados foram constituídos dois grupos, o de idosos, com idade acima de 60 anos e o grupo de não idosos, com idade entre 16 e 59 anos. Foram critérios de exclusão do estudo: histórico de hospitalização ou de uso de medicamento psiquiátrico, tratamento para hipo ou hipertireoidismo e percepção de saúde como má. Esses critérios foram estabelecidos a fim de formar uma amostra de participantes saudáveis e com características homogêneas. Os dados sociodemográficos são apresentados na Tabela 1 .

Tabela 1. Dados sociodemográficos das amostras

\begin{tabular}{llcc}
\hline & & Amostra geral & Amostra de idosos \\
\hline $\mathrm{N}$ & & 642 & 38 \\
Idade $(\mathrm{M} \pm \mathrm{DP})$ & & $26,62 \pm 13,89$ & $69,03 \pm 5,28$ \\
Anos de educação formal $(\mathrm{M} \pm \mathrm{DP})$ & & $13,82 \pm 3,02$ & $8,16 \pm 2,34$ \\
Gênero (\%) & Feminino & 63 & 89,5 \\
& Masculino & 37 & 10,5 \\
Estado Civil (\%) & Solteiro(a) & 77,9 & 13,2 \\
& Casado(a) & 13,5 & 23,7 \\
& Separado(a) & 2,7 & 15,8 \\
& Viúvo(a) & 3,6 & 42,1 \\
& Outro & 2,3 & 5,3 \\
Percepção de saúde (\%) & Boa & 87,9 & 54,1 \\
& Regular & 12,1 & 45,9 \\
\hline
\end{tabular}

Nota: N: Número de participantes. M: Média. DP: Desvio Padrão.

\section{Instrumentos}

Os participantes receberam uma Ficha de Dados Sociodemográficos, a versão em português do Questionário de Memória Prospectiva e Retrospectiva (PRMQ-10) (Benites \& Gomes, prelo; Smith \& cols., 2000) e a Escala de Auto-Eficácia Geral Percebida (EAEGP) (Scholz \& cols., 2002; Teixeira \& Dias, 2005). A Ficha de Dados Sociodemográficos foi elaborada a fim de caracterizar a população. Essa ficha forneceu informações sobre idade, sexo, anos de educação formal e estado de saúde geral. A versão em português do Questionário de Memória Prospectiva e Retrospectiva (PRMQ-10) avalia o auto-relato de falhas de memória prospectiva e retrospectiva através de 10 perguntas referentes a situações de uso cotidiano da memória. Dos 10 itens, cinco referem-se à memória prospectiva e cinco à memória retrospectiva. Cada item é seguido de uma escala Likert de cinco pontos: (1) nunca, (2) raramente, (3) algumas vezes, (4) freqüentemente e (5) quase sempre. O escore má- ximo é 50 pontos, refletindo um alto índice de queixas de memória, e o escore mínimo é 10, para um baixo índice de queixas de memória. $\mathrm{O}$ instrumento em português apresentou fidedignidade $(\alpha=0,80$, no PRMQ geral com dez itens; $\alpha=0,74$, na escala prospectiva e $\alpha=0,68$, na escala retrospectiva), validades de construto, convergente e discriminante (Benites \& Gomes, 2006). A Escala de Auto-Eficácia Geral Percebida (EAEGP) (Scholz \& cols., 2002; Teixeira \& Dias, 2005) consta de 10 itens sobre opiniões que as pessoas podem ter a respeito de si mesmas. Um item típico desta escala é: "Se estou com problemas, geralmente encontro uma saída" (item 1). Cada frase deve ser avaliada de 1 , para nem um pouco verdadeiro, até 4 , para exatamente verdadeiro. $\mathrm{O}$ número máximo de pontos é 44 e traduz uma auto-eficácia geral alta; o escore mínimo obtido através da escala é $11 \mathrm{e}$ reflete uma auto-eficácia geral baixa. Testes de validade da EAEGP em uma amostra brasileira sugeriram a unidimensionalidade do construto auto-eficácia ge- 
ral e boa consistência interna da escala $(\alpha=0,81)$ (Teixeira \& Dias, 2005).

\section{Delineamento e procedimento}

Este é um estudo de corte transversal e delineamento correlacional, cujos participantes foram contatados em uma universidade particular e em grupos de convivência. Os instrumentos foram aplicados em sessões únicas, coletivas, com duração de uma hora em média.

Ao início de cada encontro, o Termo de Consentimento Livre e Esclarecido foi lido e explicado. Logo após, os participantes tomaram conhecimento dos objetivos da pesquisa e receberam orientações quanto ao preenchimento dos instrumentos. O PRMQ-10 foi descrito como um conjunto de questões sobre falhas de memória do cotidiano comuns a todas as pessoas. Por sua vez, a EAEGP (Scholz \& cols., 2002; Teixeira \& Dias, 2005) foi descrita como um conjunto de questões sobre a percepção de si mesmo na maior parte do tempo.

Os dados foram analisados através de regressão múltipla, usando-se escores de auto-relato de falhas de memória geral, prospectiva e retrospectiva do PRMQ10 como variáveis dependentes, analisados um de cada vez e escores da EAEGF como variáveis independentes. Análises de covariância foram também realizadas com a adição de variáveis categóricas binárias (idoso/não idoso e baixo/alto nível de autoeficácia) em análises de regressão múltipla.

O estudo foi previamente aprovado pelo Comitê de Ética em Pesquisa da Universidade Federal do Rio Grande do Sul (CEP/UFRGS No 2005425). Foram seguidas as Diretrizes e Normas Regulamentadoras de Pesquisas Envolvendo Seres Humanos, do Conselho Nacional de Saúde (CNS), conforme resolução 196/96 e do Conselho Federal de Psicologia (CFP), conforme resolução 016/2000. Todos os participantes deram seu consentimento por escrito após serem esclarecidos sobre os procedimentos da pesquisa.

\section{RESULTADOS}

Foram realizadas três análises em separado, nas quais as variáveis independentes (auto-eficácia geral, idade e anos de escolaridade) foram adicionadas aos modelos segundo o grau de correlação com a variável dependente (auto-relato de falhas de memória geral, prospectiva e retrospectiva). A análise de regressão hierárquica foi realizada a fim de determinar se idade e anos de educação melhoravam a predição do auto- relato de falhas de memória (geral, prospectiva e retrospectiva), predita pelo escore de auto-eficácia geral. Os dados da medida de auto-relato de falhas de memória (PRMQ-10) sofreram sofreram transformação raiz quadrada, a fim de melhorar a normalidade, linearidade e homocedasticidade dos resíduos.

Após a entrada de todas as variáveis, elas explicaram $9 \%$ do auto-relato de falhas de memória em geral; $6 \%$ do auto-relato de falhas de memória prospectiva e $8 \%$ do auto-relato de falhas de memória retrospectiva. $\mathrm{Na}$ análise do auto-relato de falhas de memória em geral, após a primeira fase, auto-eficácia geral explicou $6,1 \%$, a adição da idade explicou $8,6 \%$ e a adição de anos de escolaridade não resultou em um incremento significativo $(p>0,05)$. Na análise do autorelato de falhas de memória prospectiva, a adição da variável auto-eficácia na equação resultou em uma explicação de $4,9 \%$, a adição de idade explicou $5,7 \%$ e a adição de escolaridade novamente não foi significativa $(\mathrm{p}>0,05)$ para a mudança do $\mathrm{F}$. Na análise do auto-relato de falhas de memória retrospectiva, autoeficácia geral explicou $4,4 \%$ das falhas retrospectivas auto-relatadas, a adição das variáveis idade e escolaridade explicou 7,8\% e 8,3\%, respectivamente (Tabela 2).

A análise de covariância do auto-relato de falhas de memória entre grupo de idosos e não idosos foi realizada através da inclusão da variável qualitativa binária "grupo etário" na análise de regressão múltipla. Sendo que idosos tendem a ter auto-eficácia mais baixa do que adultos jovens (Hertzog \& cols., 1987; Levy, 1996), o nível de auto-eficácia geral foi utilizado como covariável nessas análises. Pode-se, então, investigar estritamente a variação do auto-relato de falhas em idosos e não idosos, sem a mediação das variações da auto-eficácia geral. As equações encontradas foram: para o relato de falhas de memória em geral $\left(R^{2}=0,07, p<0,01\right), y$ (idosos) $=(17,7+0,6=$ $18,3)-0,1 \mathrm{x}$ e y (não idosos) $=17,6-0,1 \mathrm{x}$. Para o relato de falhas de memória prospectiva $\left(\mathrm{R}^{2}=0,06\right.$, $\mathrm{p}>0,05), y($ idosos $)=9,5-0,05 x$ e y $($ não idosos $)=$ $9,3-0,05 x$. Para o relato de falhas de memória retrospectiva $\left(\mathrm{R}^{2}=0,06, \mathrm{p}<0,01\right)$, y (idosos) $=8,8-$ $0,05 x$ e y (não idosos) $=8,3-0,05 x$ (Tabela 3 ). Os resultados demonstram haver uma diferença estatisticamente significativa entre idosos e não idosos quanto ao auto-relato de falhas de memória geral e retrospectiva, não se encontrando diferença significativa quanto às falhas de memória prospectiva. $\mathrm{O}$ grupo de idosos relatou significativamente mais falhas de memória geral $(B=+0,64)$ e retrospectiva $(B=+0,48)$ do que o grupo de não idosos. 
Tabela 2. Variabilidade explicada $\left(\mathrm{R}^{2}\right)$ e coeficientes de regressão $(B)$ obtidos na última análise dos escores (transformados) de auto-relato de falhas de memória, na amostra total $(\mathrm{N}=642)$

\begin{tabular}{|c|c|c|c|c|c|}
\hline \multirow[b]{2}{*}{ Ordem de entrada das variáveis } & \multirow[b]{2}{*}{$\mathbf{R}$} & \multirow[b]{2}{*}{$R^{2}$ ajustado } & \multicolumn{2}{|c|}{$\begin{array}{l}\text { Coeficientes parciais de } \\
\text { regressão* }\end{array}$} & \multirow[b]{2}{*}{$p$} \\
\hline & & & B & $\beta$ & \\
\hline \multicolumn{6}{|c|}{ Auto-relato de falhas de memória em geral } \\
\hline 1. Auto-eficácia geral & $0,251^{\mathrm{a}}$ & 0,061 & $-0,10$ & $-0,26$ &, 000 \\
\hline 2. Idade & $0,299 b$ & 0,086 & 0,02 & 0,15 &, 000 \\
\hline 3. Anos de educação & $0,306 \mathrm{c}$ & 0,089 & $-0,04$ & $-0,06$ & ,105 \\
\hline (Constante) & & & 17,85 & &, 000 \\
\hline \multicolumn{6}{|c|}{ Auto-relato de falhas de memória prospectiva } \\
\hline 1. Auto-eficácia geral & $0,226^{\underline{a}}$ & 0,049 & $-0,05$ & $-0,23$ &, 000 \\
\hline 2. Idade & $0,246 b$ & 0,057 & 0,007 & 0,09 & ,032 \\
\hline 3. Anos de educação & $0,247 c$ & 0,056 & $-0,009$ & $-0,03$ &, 532 \\
\hline (Constante) & & & 9,24 & &, 000 \\
\hline \multicolumn{6}{|c|}{ Auto-relato de falhas de memória retrospectiva } \\
\hline 1. Auto-eficácia geral & $0,214^{a}$ & 0,044 & $-0,05$ & $-0,22$ &, 000 \\
\hline 2. Idade & $0,285 b$ & 0,078 & 0,01 & 0,17 &, 000 \\
\hline 3. Anos de educação & $0,296 c$ & 0,083 & $-0,03$ & $-0,08$ &, 049 \\
\hline (Constante) & & & 8,56 & &, 000 \\
\hline
\end{tabular}

* Coeficientes parciais de regressão obtidos na última análise.

a. Preditores: (constante), auto-eficácia geral.

b. Preditores: (constante), auto-eficácia geral, idade.

c. Preditores: (constante), auto-eficácia geral, idade, anos de educação.

Tabela 3. Análise de covariância do auto-relato de falhas de memória em grupos de idosos e não idosos ( $N=642)$

\begin{tabular}{lcccc}
\hline & $\mathbf{R}$ & $\mathbf{R}^{2}$ ajustado & $\mathbf{B}$ & $\mathbf{p}$ \\
\hline Auto-relato de falhas de memória em geral & & & & \\
Auto-eficácia geral & & & $-0,10$ &, 000 \\
Idosos/ não idosos & 0,277 & 0,074 & 0,64 &, 028 \\
(Constante) & & & 17,65 &, 000 \\
\hline Auto-relato de falhas de memória prospectiva & & & \\
Auto-eficácia geral & & $-0,5$ &, 000 \\
Idosos/ não idosos & 0,254 & 0,061 & 0,15 &, 347 \\
(Constante) & & & 9,36 &, 000 \\
\hline Auto-relato de falhas de memória retrospectiva & & & \\
Auto-eficácia geral & & & 0,05 &, 000 \\
Idosos/ não idosos & 0,240 & 0,055 & 0,48 &, 004 \\
(Constante) & & & 8,29 &, 000 \\
\hline
\end{tabular}

Para a análise da variância do auto-relato de falhas de memória nos grupos de auto-eficácia baixa (até 33 pontos na EAEGP) e alta (34 pontos e acima na EAEGP), a variável binária nível de auto-eficácia (alta/baixa) foi adicionada à análise de regressão múltipla, usando-se a idade como covariável. As equações foram: para auto-relato de falhas de memória em geral $\left(\mathrm{R}^{2}=0,07, \mathrm{p}<0,01\right)$, y (auto-eficácia baixa) $=14,2-$ $0,02 \mathrm{x}$ e y (auto-eficácia alta) $=13,4+0,02 \mathrm{x}$; para auto-relato de falhas de memória prospectiva $\left(\mathrm{R}^{2}=\right.$ $0,05, \mathrm{p}<0,001)$, y (auto-eficácia baixa) $=7,6+0,008 \mathrm{x}$ e y(auto-eficácia alta $)=7,2+0,02 x$; para o auto-relato de falhas de memória retrospectiva $\left(\mathrm{R}^{2}=0,07\right.$, $\mathrm{p}<0,01$ ), y (auto-eficácia baixa) $=6,6+0,02 x$ e y (autoeficácia alta) $=6,2+0,02 x$ (Tabela 4). Observa-se que, controlando pela idade, pessoas com auto-eficácia alta têm menos auto-relato de falhas de memória geral (B $=-0,86)$ do que pessoas com auto-eficácia baixa. 
Tabela 4. Análise de covariância do auto-relato de falhas de memória em grupos de auto-eficácia geral baixa e alta corrigido pela idade $(\mathrm{N}=642)$

\begin{tabular}{|c|c|c|c|c|}
\hline & $\mathbf{R}$ & $\mathbf{R}^{2}$ ajustado & B & $p$ \\
\hline \multicolumn{5}{|c|}{ Auto-relato de falhas de memória em geral } \\
\hline Idade & & & 0,02 &, 000 \\
\hline Auto-eficácia geral alta / baixa & 0,270 & 0,070 & $-0,86$ &, 000 \\
\hline (Constante) & & & 14,22 &, 000 \\
\hline \multicolumn{5}{|c|}{ Auto-relato de falhas de memória prospectiva } \\
\hline Idade & & & 0,01 &, 000 \\
\hline Auto-eficácia geral alta / baixa & 0,217 & 0,044 & $-0,41$ & 009 \\
\hline (Constante) & & & 7,59 &, 000 \\
\hline \multicolumn{5}{|c|}{ Auto-relato de falhas de memória retrospectiva } \\
\hline Idade & & & 0,02 &, 000 \\
\hline Auto-eficácia geral alta / baixa & 0,263 & 0,066 & $-0,44$ &, 000 \\
\hline (Constante) & & & 6,63 &, 000 \\
\hline
\end{tabular}

A fim de analisar o efeito da idade nos resultados descritos acima, a mesma análise foi repetida sem a introdução dessa variável. $\mathrm{O}$ escore médio de falhas de memória em geral aumentou 0,75 unidades do grupo de auto-eficácia geral baixa para o grupo de autoeficácia geral alta $\left(\mathrm{R}^{2}=0,04, \mathrm{p}<0,01\right)$. O acréscimo é de 0,38 unidades ao auto-relato de falhas de memória prospectiva $\left(\mathrm{R}^{2}=0,03, \mathrm{p}<0,01\right)$ e de 0,37 unidades na média do auto-relato de falhas de memória retrospectiva $\left(R^{2}=0,03, p<0,01\right)$. Tais resultados demonstram a importância de levar em consideração a idade quando se avalia o auto-relato de falhas de memória.

\section{DISCUSSÃO}

A análise de fatores que poderiam contribuir para o auto-relato de falhas de memória verificou a reciprocidade entre características do auto-relato e resultados de desempenho de memória presentes na literatura. A contribuição exclusiva da escolaridade para a predição de desempenho de memória retrospectiva encontrada experimentalmente por Comijs e cols. (2000) foi verificada de modo análogo neste estudo. Escolaridade explicou significativamente o auto-relato de falhas de memória retrospectiva e não explicou o auto-relato de falhas de memória prospectiva.

A variável idade explicou os três tipos de autorelato de falhas de memória, sendo menos representativa para o auto-relato de falhas de memória prospectiva, segundo a análise de regressão hierárquica. Nas análises de variância, o auto-relato de falhas de memória prospectiva não mudou significativamente entre os grupos de idosos e não idosos. Tais resultados apontam que, apesar das falhas prospectivas precede- rem as falhas retrospectivas no envelhecimento normal (McDaniel \& Einstein, 2000), queixas prospectivas podem ser disfarçadas no cotidiano através do uso de pistas e serem menos relatadas do que as queixas retrospectivas.

Resultados da associação entre idade, memória prospectiva e retrospectiva diferem de acordo com o método de investigação: experimental ou naturalístico. Em testes experimentais idosos tendem a apresentar resultados inferiores aos adultos e o padrão contrário é encontrado em pesquisas naturalísticas. Os resultados desta pesquisa apresentam maior congruência com os resultados de estudos naturalísticos, nos quais idosos fazem maior uso de estratégias para a lembrança prospectiva (Henry, MacLeod, Phillips \& Crawford, 2004). Portanto, o auto-relato de falhas de memória prospectiva parece estar mais fortemente vinculado a características pessoais, como aponta a associação positiva e significativa entre o auto-relato prospectivo e auto-eficácia geral.

A auto-eficácia geral é o conceito central da teoria sócio-cognitiva de Bandura. Nesta, intencionalidade e antecipação estão entre as principais capacidades que medeiam o comportamento humano (Bandura, 2001). Essas características encontram-se teoricamente relacionadas com a memória prospectiva. Segundo a visão de multiprocessos (McDaniel \& Einstein, 2000), o tipo de intenção influencia a lembrança e/ou falha da recuperação da memória prospectiva. Ainda, processos cognitivos poderiam ser específicos para cada tipo de intenção, a qual parece possuir um limiar reduzido, ou um nível de ativação aumentado, quando é induzida por pistas externas (Einstein, Smith, McDaniel \& Shaw, 1997). 
O fato de ser ou não idoso explica $8 \%$ da variação no auto-relato de falhas de memória em geral. A média nesse tipo de auto-relato de falhas aumentou 0,64 unidades no grupo de pessoas com 60 anos ou mais, comparando-os com o grupo de idade inferior a 60 anos. $\mathrm{O}$ auto-relato de falhas de memória retrospectiva variou até $6 \%$ entre os participantes que pertenciam ao grupo de idosos e os não idosos. A média desse autorelato aumentou 0,48 unidades do grupo de não idosos para o grupo de idosos. Este resultado demonstra uma maior associação entre idade e auto-relato de falhas de memória retrospectiva e a falta de associação entre idade e auto-relato de falhas de memória prospectiva, resultado recíproco ao estudo de Henry e cols. (2004). Esta pesquisa apresentou evidência de que o uso da memória prospectiva no cotidiano pode não estar associado à idade devido ao provável uso de pistas pelos idosos. Henry e cols. (2004) realizaram uma tarefa prospectiva, na qual idosos e adultos jovens deveriam realizar uma ligação telefônica para o laboratório da pesquisa após dois dias do primeiro contato. A finalização satisfatória da tarefa por um maior número de idosos no experimento foi vinculada à motivação e ao uso de pistas pelos idosos. Ainda, a confiança dos idosos no uso de pistas pode revelar experiência anterior de falhas de memória.

A variável auto-eficácia geral contribuiu significativamente para a predição do auto-relato de falhas de memória em geral $(6,3 \%)$, prospectiva $(5,1 \%)$ e retrospectiva $(4,6 \%)$. A interferência da auto-eficácia geral foi maior no auto-relato de falhas de memória prospectiva do que no auto-relato de falhas retrospectivas, resultado análogo ao de McDonald, Gould e Tychynski (1999). Nesse experimento, os autores examinaram a relação entre auto-eficácia de memória, e desempenho de memória prospectiva e retrospectiva em 50 adultos maduros. Os resultados indicaram que a auto-eficácia de memória influenciava significativamente o desempenho da memória prospectiva, mas não da memória retrospectiva.

A análise da variância do auto-relato de falhas de memória em grupos de auto-eficácia (baixa/alta) revelou que a média do auto-relato de falhas de memória em geral aumenta 0,75 unidades do grupo com baixa auto-eficácia para o grupo com alta autoeficácia geral. Esse índice é de 0,38 para o auto-relato de falhas de memória prospectiva e 0,37 para o autorelato de falhas retrospectivas. Porém, quando idade foi adicionada ao modelo como controle, o nível de auto-eficácia passou a influenciar mais o auto-relato de falhas de memória retrospectiva (aumento de 0,44 unidades na média de memória retrospectiva e 0,41 na prospectiva). Ou seja, o auto-relato de falhas de memória retrospectiva está mais fortemente vinculado à idade e é influenciado secundariamente pela autoeficácia geral.

\section{CONCLUSÕES}

Os presentes achados apontam para um provável padrão na relação entre os construtos auto-eficácia e memória, no qual auto-eficácia de memória está mais fortemente associada ao desempenho prospectivo e auto-eficácia geral ao auto-relato de falhas de memória prospectiva. As dimensões de auto-eficácia e memória prospectiva podem apresentar maior correlação por estarem vinculadas à expectativa e à formação, e execução de planejamentos (Bandura, 1989; 2001; Crawford \& cols., 2003; Smith \& cols., 2000). Quanto à dimensão retrospectiva, escolaridade e idade apresentaram maior associação com auto-relato de falhas, e com o desempenho de memória retrospectiva (Comijs \& cols., 2000; Henry \& cols., 2004). Sendo assim, uma maior escolaridade tende a predizer tanto um melhor desempenho em tarefas de memória retrospectiva, quanto um menor auto-relato de falhas de memória retrospectiva. Com relação à idade, mais anos de vida tende a predizer um menor desempenho em tarefas retrospectivas e um maior número de autorelato de falhas de memória retrospectiva. Ressalta-se, contudo, que tais considerações têm como base um grupo de idosos formado basicamente por mulheres.

O manejo da auto-eficácia geral tanto individualmente quanto coletivamente, através da modificação de estereótipos sociais (Jang, Poon, Kim \& Shin, 2004), pode diminuir diretamente o auto-relato de falhas de memória prospectiva e indiretamente o autorelato de falhas de memória retrospectiva. Numa direção contrária, a diminuição de queixas tende a aumentar a auto-eficácia geral e a percepção de qualidade de vida (Borglin, Edberg \& Hallberg, 2005). Os resultados da pesquisa apresentaram pontos de reciprocidade com resultados de desempenho de memória em tarefas prospectivas e retrospectivas. Desse modo, pode-se concluir que os auto-relatos de falhas de memória prospectiva e retrospectiva são confiáveis e podem ser utilizados em triagens e em baterias de avaliação cognitiva. A atenção e a avaliação do autorelato de falhas de memória prospectiva em idosos, logo no início de possíveis quadros patológicos, pode propiciar melhores resultados no tratamento. Com efeito, o auto-relato de falhas de memória prospectiva 
em idosos geralmente antecede as queixas de memória retrospectiva.

Supõe-se que o estresse é um fator emocional cuja predominância em certos períodos na vida do adulto jovem pode elevar o auto-relato de falhas de memória. Entre idosos, falhas de memória prospectiva ou retrospectiva podem ser mais relatadas devido à presença de sintomas depressivos. A investigação da associação entre estados emocionais e auto-relato de falhas de memória prospectiva, principalmente, pode trazer dados que melhor caracterizem diferenças e semelhanças entre as dimensões prospectiva e retrospectiva de memória. Os resultados também corroboram o uso do PRMQ-10 (Smith \& cols., 2000) para o estudo do auto-relato de falhas de memória prospectiva e retrospectiva em contraste com múltiplos aspectos emocionais e de personalidade.

\section{REFERÊNCIAS}

Bandura, A. (2001). Social cognitive theory: an agentic perspective. Annual Review of Psychology, 52, 1-26.

Bandura, A. (1998). Health promotion from the perspective of social cognitive theory. Psychology and Health, 13, 623-649.

Bandura, A. (1994). Self-efficacy. Em V. S. Ramachaudran (Org.), Encyclopedia of human behavior (pp. 71-81). New York: Academic Press.

Bandura, A. (1989). Regulation of cognitive processes through perceived self-efficacy. Developmental Psychology, 25, 725739

Bandura, A. (1977). Self-efficacy: toward a unifying theory of behavioral change. Psyhcological Review, 89, 191-215.

Bandura, A.; Barbaranelli, C., Caprara, G. V. \& Pastorelli, C. (2001). Self-efficacy beliefs as shapers of children's aspirations and career trajectories. Child Development, 72, 187-206.

Bandura, A. \& Locke, E. (2003). Negative self-efficacy and goal effects revisited. Journal of Applied Psychology, 88, 87-99.

Berry, J. M., West, R. L. \& Dennehey, D. M. (1989). Reliability and validity of the Memory Self-Efficacy Questionnaire. Developmental Psychology, 25 (5), 701-713.

Benites, D. \& Gomes, W. B. (2006). Características psicométricas do Prospective and Retrospective Memory Questionnaire (PRMQ) Psico USF.

Borglin, G., Edberg, A-K. \& Hallberg, I. R. (2005). The experience of quality of life among old people. Journal of Aging Studies, 19, 201-220.

Carneiro, R. S. \& Falcone, E. M. O. (2004). Um estudo das capacidades e deficiências em habilidades sociais na terceira idade. Psicologia em Estudo, 9 (1), 119-126.

Cavanaugh, J. C. \& Green, E. E. (1990). I believe, therefore I can: Self-efficacy beliefs in memory aging. Em E. A. Lovelace (Org.), Aging and cognition: mental processes, self awareness, and interventions (pp. 189-230). Amsterdam: Elsevier.

Comijs, H. C., Deeg, D. J. H.; Dik, M. G., Twisk, J. W. R. \& Jonker, C. (2002). Memory complaints: the association with psycho-affective and health problems and the role of persona- lity characteristics. Journal of Affective Disorders, 72, 157165.

Crawford, J., Smith, G., Maylor, E. A., Sala, S. D. \& Logie, R. (2003). The Prospective and Retrospective Memory Questionnaire (PRMQ): normative data and latent structure in a large non-clinical sample. Memory, 11 (3), 261-275.

Eccles, J. S. \& Wigfield, A. (2002). Motivational beliefs, values, and goals. Annual Review of Psychology, 53, 109-132.

Einstein, G. O., Smith, R. E., McDaniel, M. A. \& Shaw, P. (1997). Aging and prospective memory: the influence of increased task demands at encoding and retrieval. Psychology and Aging, 12, 479-488.

Henry, J. D., MacLeod, M. S., Phillips, L. H. \& Crawford, J. R. (2004). A meta-analytic review of prospective memory and aging. Psychology \& Aging, 19 (1), 27-39.

Hertzog, C., Dixon, R. A., Schulenberg, J. E. \& Hultsch, D. (1987). On the differentiation of memory beliefs from memory knowledge: the factor structure of the metamemory in adulthood scale. Experimental Aging Research, 13 (2), 101-107.

Jang, Y., Poon, L. W., Kim, S-Y \& Shin, B-K. (2004). Selfperception of aging and health among older adults in Korea. Journal of Aging Studies, 18, 485-496.

Levy, B. (1996). Improving memory in old age through implicit self-stereotyping. Journal of Personality and Social Psychology, 71 (6), 1092-1107.

McDaniel, M. A. \& Einstein, G. O. (2000). Strategic and automatic processes in prospective memory retrieval: a multiprocess framework. Applied Cognitive Psychology, 14, 127-144.

Marsh, R., L., Hicks, J. L. \& Watson, V. (2002). The dynamics of intention retrieval and coordination of action in event-based in prospective memory. Journal of Experimental Psychology: Learning, Memory and Cognition, 28 (4), 652-659.

McDonald, M. L., Gould, O. N. \& Tychynski, D. (1999). Metamemory predictors of prospective and retrospective memory performance. Journal of General Psychology, 126 (1), 3752.

McDougall, G. J. \& Kang, J. (2003). Memory self-efficacy and memory performance in older males. International Journal of Men's Health, May, 2003.

Sherer, M. \& Maddux, J. E. (1982). The self-efficacy scale: construction and validation. Psychological Reports, 51, 663671.

Scholz, U., Doña, B. G., Sud, S. \& Schwarzer, R. (2002). Is general self-efficacy a universal construct? Psychometric findings from 25 countries. European Journal of Psychological Assessment, 18 (3), 242-251.

Schröder, K. E. E., Schwarzer, R. \& Konertz, W. (1998). Coping as a mediator in recovery from cardiac surgery. Psychology \& Health, 13, 83-97.

Schwarzer, R., Hahn, A. \& Jerusalem, M. (1993). Negative affect in East German migrants: longitudinal effects of unemployment and social support. Anxiety, Stress, Coping, 6, 57-69.

Seeman, T. E., Rodin J. \& Albert, M. A. (1993). Self-efficacy and cognitive performance in higher functioning older individuals: MacArthur Studies of Successful Aging. Journal of Aging and Health, 5, 455-474.

Smith, G., Sala, S. D., Logie, R. \& Maylor, E. A. (2000). Prospective and retrospective memory in normal aging and dementia: a questionnaire study. Memory, 8 (5), 311-321. 
Teixeira, M. A. P. \& Dias, A. C. G. (2005). Propriedades psicométricas da versão traduzida para o português da Escala de Auto-eficácia Geral Percebida de Ralph Schwarzer. [Resumo]. Em Congresso Brasileiro de Avaliação Psicológica. Resumos do II Congresso Brasileiro de Avaliação Psicológica (CDROM). Gramado, RS.

Telch, M. J. \& Bandura, A. (1982). Social demand for consistency and congruence between self-efficacy and performance. Behavior Therapy, 13, 694-701.
Welch, D. C. \& West, R. L. (2005). Self-efficacy and mastery: its application to issues of environmental control, cognition and aging. Developmental Review, 15, 150-171.

Recebido: 25/08/2006

Revisado: $15 / 12 / 2006$ Aceito: 30/12/2006

Nota:

${ }^{1}$ Pesquisa realizada com apoio da CAPES.

\section{Sobre os autores:}

Daniela Benites concluiu o Curso de Psicologia na Universidade Regional Integrada - Campus de Santo Angelo - RS, ingressando a seguir no Programa de Pós-Graduação em Psicologia da Universidade Federal do Rio Grande do Sul, onde concluiu o Mestrado e cursa atualmente o doutorado sob orientação de W. B. Gomes. O presente trabalho faz parte de sua dissertação de Mestrado.

Sidia M. Callegari Jacques é doutora em Genética pela UFRGS e professora titular do Departamento de Estatística da UFRGS, tendo ativa participação em ensino e pesquisa nos programas de pós-graduação em Genética e Biologia Molecular e em Medicina, ambos da UFRGS. É autora, entre outros, do livro Bioestatística: princípios e aplicações, pela Artmed.

Gustavo Gauer é psicólogo e doutor em psicologia pela UFRGS, tendo desenvolvido projeto de Doutorado Sanduíche sobre memória, na Duke University. Atualmente é professor de Psicologia na UFMG.

William B. Gomes faz parte do grupo fundador do Programa de Pós-Graduação em Psicologia da Universidade Federal do Rio Grande do Sul, onde leciona disciplinas relacionadas à Epistemologia e História da Psicologia, e coordenador do Laboratório de Cognição e Fenomenologia Experimental. Foi também fundador e primeiro editor da Revista Psicologia: Reflexão e Crítica. É bolsista de produtividade $1 \mathrm{~A}$ do CNPq.

Endereço para correspondência: William B. Gomes - Rua Gen. Couto de Magalhães, 1.155/601 - 90540-131 Porto Alegre - RS. Endereço eletrônico: gomesw@ufrgs.br. 